\title{
Understanding the contributions of visual stimuli to contextual fear conditioning: A proof-of-concept study using LCD screens
}

\author{
Nathen J. Murawski ${ }^{\mathrm{a},{ }^{\star}, 1}$, Arun Asok ${ }^{\mathrm{b}, \mathrm{c}, 1}$ \\ ${ }^{a}$ Center for Brain Science, Harvard University, Cambridge, MA 02138, United States \\ ${ }^{b}$ Department of Neuroscience, Columbia University, New York, NY 10032, United States \\ 'Howard Hughes Medical Institute, New York, NY 10032, United States
}

\section{Abstract}

The precise contribution of visual information to contextual fear learning and discrimination has remained elusive. To better understand this contribution, we coupled the context pre-exposure facilitation effect (CPFE) fear conditioning paradigm with presentations of distinct visual scenes displayed on 4 LCD screens surrounding a conditioning chamber. Adult male Long-Evans rats received non-reinforced context pre-exposure on Day 1, an immediate $1.5 \mathrm{~mA}$ foot shock on Day 2, and a non-reinforced context test on Day 3. Rats were pre-exposed to either digital Context (dCtx) A, dCtx B, a distinct Ctx C, or no context on Day 1. Digital context A and B were identical except for the visual image displayed on the LCD screens. Immediate shock and retention testing occurred in dCtx A. Rats pre-exposed dCtx A showed the CPFE with significantly higher levels of freezing compared to controls. Rats pre-exposed to Context B failed to show the CPFE, with freezing that did not highly differ from controls. These results suggest that visual information contributes to contextual fear learning and that visual components of the context can be manipulated via LCD screens. Our approach offers a simple modification to contextual fear conditioning paradigms whereby the visual features of a context can be manipulated to better understand the factors that contribute to contextual fear discrimination and generalization.

\section{Keywords}

Contextual fear conditioning; CPFE; Fear conditioning; Visual features; Visual elements; Learning and memory

\section{Introduction}

\begin{abstract}
In fear conditioning, manipulating contextual cues is important for elucidating how learning is either "context-specific" or "context-independent" given that distinct, although possibly overlapping [6,29], neural systems support different types of conditioned fear [13,20]. "Context," in fear conditioning, is defined as the multimodal sensory experience, including
\end{abstract}

\footnotetext{
${ }^{1}$ These authors contributed equally to this work. This work appears as a pre-print, submitted to BioRxivon 8/15/2016.

*Corresponding author. Present/Permanent address: Harvard University, Fas Center for Brain Science, Northwest Lab Building, 52 Oxford Street, Cambridge, MA 02138, United States. nathenmurawski@gmail.com (N.J. Murawski).
} 
temporal and spatial factors, encountered concurrently during a conditioning trial (for reviews see [17,31,36]; the context is usually the conditioning chamber, an enclosed box with visual, tactile, olfactory, and auditory cues that are incidentally encountered and associated with an aversive stimulus (e.g., foot shock). Following conditioning, rodents typically exhibit freezing (a species-specific defensive reaction; [2,3]) when placed back into the context where the foot shock occurred, but not in a novel context, suggesting that the defensive reaction is context specific. However, the features that make one context distinct from another are unclear. Typically, researchers manipulate multiple sensory features (e.g., odors, lighting, spatial layout, etc.) of a context to make one context distinct from another. Yet, our understanding of how the independent sensory features of a context differentially contribute to the CS-US representation (i.e., is one feature more prominent than another?) or what specific elements are most important in distinguishing between contexts is limited [10].

Rodents are able to utilize visual information presented on LCD screens to perform a variety of behavioral tasks such as navigating a virtual maze or making appropriate behavioral choices [21,38,39]. Additionally, presenting looming or sweeping visual stimuli on a ceilingmounted LCD screen - to simulate predatory threats - can control rodent flight or freezing behavior [8]. Incorporating LCD monitors into contextual fear conditioning paradigms may offer a means to systematically control visual elements of a context during aversive learning.

We used a variant of contextual fear conditioning known as the context pre-exposure facilitation effect (CPFE) paradigm. The CPFE paradigm separates incidental contextual learning from context-shock associative learning. The CPFE relies on the immediate shock deficit - a phenomenon where animals that are not given enough time to learn about the context prior to receiving a shock (e.g., <10-s [9]) fail to exhibit conditioned freezing [5,11]. However, pre-exposure to the conditioning context on the day prior to receiving an immediate shock is sufficient to overcome this deficit [11]. More importantly, the CPFE is only evident when context pre-exposure occurs to the training context and not a distinct context [33]. While the CPFE paradigm has been useful in understanding how gross incidental contextual learning is processed distinctly from fear-learning [23,24], the contribution of visual features to contextual learning remains unclear.

In the present study, we investigated how altering visual information in the CPFE affects contextual fear-learning. We restricted changes in visual features to incidental contextual learning during context pre-exposure. We placed four LCD monitors around a clear chamber (three on the sides and one on top); each displayed one of two images on all screens during the pre-exposure phase, with all other context features (i.e., tactile, spatial, olfactory, and auditory components) held constant between the two groups. During immediate-shock training and testing, only one of the two visual scenes was displayed on the monitors. We hypothesized that rats pre-exposed to the testing context would show the CPFE whereas those pre-exposed to the alternate visual context would not. We also included two control groups as CS and US associative-learning controls, one of which was pre-exposed to a distinct context (different visual, spatial, auditory, and olfactory components) and one that received no pre-exposure. 


\section{Methods}

\subsection{Subjects}

Forty adult male Long Evans rats 8-9 weeks of age were used. Thirty-two were purchased from Harlan breeders (Indianapolis, IN) and eight were bred in house (University of Delaware). All rats were housed in the animal colony at the University of Delaware. Pairs of rats were housed in opaque polypropylene cages $(45 \times 24 \times 21 \mathrm{~cm})$ with standard bedding and free access to food and water. Rats were maintained on a 12:12 h light/dark cycle with lights on at 7:00a.m. Testing occurred during the light phase (12:00P.M.-5:00P.M.). All animals were treated in accordance with NIH guidelines for the care and use of laboratory animals.

\subsection{Apparatus}

The conditioning chamber was made out of clear Plexiglas $(40 \times 22 \times 24 \mathrm{~cm})$; one of the four walls could be opened to allow for animal placement. The chamber floor consisted of 40 grid bars $(0.4 \mathrm{~cm}$ in diameter) that ran parallel to the shorter wall of the chamber. The grid bars were connected to a shock generator (Med Associates; ENV 410B) that delivered an alternating current foot shock US. Four LCD monitors (Dell, Plano, TX) were placed flush to three of the external walls of the chamber with one monitor acted as the ceiling. All monitors projected the same image concurrently and were connected to a Dell computer. The monitors projected one of two images (found on an internet search without copyright attribution; Fig. 1A). The first image consisted of multiple pumpkins with dominant ovoid contours and light and dark coloring throughout. The second image consisted of rock formations with dominant linear contours featuring darker coloring toward one side and lighter coloring towards the other side. The lighting levels between the two images were adjusted via the LCD monitors so that their total luminance ( 200-225 lx) was equivalent. Image presentation was controlled by custom software (available at: https:// sites.google.com/site/aaasok/programmed-software). The conditioning chamber was cleaned with a 70\% ethanol solution prior to animal placement. When the monitors projected the pumpkin image, the chamber was determined to be in the Context A configuration; projection of the rock formation produced the Context $\mathrm{B}$ configuration. With the exception of the projected images, there were no other differences between Context A and B.

The conditioning chamber was located in a dark room with low-levels of background noise produced by ventilation. A CCD camera (Model \# ACT-VP-02, Actimetrics, Wilmette, IL) mounted on a tripod was placed approximately $61 \mathrm{~cm}$ in front of the clear wall of the conditioning chamber. The camera was connected to a computer running FreezeFrame3 software (Actimetrics, Wilmette IL), which controlled session protocol (see below) and foot shock presentation.

A separate Plexiglas chamber $(16.5 \times 21.1 \times 21.6 \mathrm{~cm})$ was used as Context $\mathrm{C}$. This chamber was situated in a separate, dark room within a fume hood that provided ambient noise ( 74 $\mathrm{dB})$ and lighting $(\sim 12001 \times)$. The chamber had a grid floor with 9 metal bars $(0.5 \mathrm{~cm}$ in diameter and placed $1.25 \mathrm{~cm}$ apart) parallel to the long length of the chamber. One side of 
the chamber was painted white. The chamber was cleaned with a 5\% ammonium hydroxide solution prior to animal placement.

\subsection{Procedure}

2.3.1. General procedure-Four groups of rats were included: Group No Pre received no pre-exposure; Group Ctx C was pre-exposed to Context C; Group dCtx B was preexposed to digital context B; and Group dCtx A was pre-exposed to digital context A. Prior to each behavioral session (see below) rats were brought up from the animal colony in their home cages and then placed on a cage rack in a waiting room adjacent to the testing room. Rats were run one at a time, taken from their home cage and placed into a black ice bucket with the cover on and transferred to the testing room. Each rat was quickly removed from the ice bucket and placed into the conditioning chamber $(<30 \mathrm{~s}$.). At the end of the session, the rat was quickly removed from the conditioning chamber, placed back into the ice bucket, and returned to their holding cage while the experimenter cleaned out the chamber and ice bucket for the next rat.

2.3.2. Behavioral procedure (Fig. 1B)—Fig. 1 provides a schematic of the behavioral testing procedure, which included three sessions, each 24-h apart.

For context pre-exposure, rats were pre-exposed to one of three contexts ( $\mathrm{dCtx} \mathrm{A}$ or B or Ctx C). A fourth group was briefly removed and returned to their home cage to equate handling (No Pre). Preexposure consisted of a 300-s session in the absence of any US presentation. To match the tactile experience across digital contexts, a dark grey Plexiglas sheet was placed over the grid floor.

For immediate shock training, rats were placed into digital Context $\mathrm{A}$ and received an immediate ( $<5$-s) $1.5 \mathrm{~mA}, 2$-s AC foot shock US. Rats were removed from the conditioning chamber within 5-s of foot shock presentation.

For context testing, rats were placed in digital Context $\mathrm{A}$ and their freezing behavior was monitored over a 300-s testing session. Prior to placement, the dark grey Plexiglas floor covering (that was used during pre-exposure) was placed over the grid floor.

\subsection{Data analysis}

2.4.1. Freezing analyses-The data were collected and scored using FreezeFrame3 software (Actimetrics, Wilmette IL). The bout length was set to 0.75-s and the freezing threshold (changes in pixels per frame) was adjusted by an experimenter so that no small movements were registered as freezing.

2.4.2. Statistical analyses-SPSS 23.0 was used for all data analyses (IBM Corp, Armonk, NY). Following analysis for homoscedasticity, freezing behavior during preexposure and testing sessions was analyzed separately with a repeated-measures (RM) ANOVA across five 60-s time bins. Time bin was included as the within-subject's variable and group (Preexposure context) as the between-subject's variable. We used this design because we were interested how freezing changed over time within sessions between groups. We did not use an RM-ANOVA to analyze the overall change in freezing from pre-exposure 
to testing because the No Pre group was not pre-exposed to any context and thus had no preexposure data. At each time point, post-hoc simple effects analyses were used to compare differences between groups following significant interactions. Post-hoc Dunnet's 2-sided contrasts were also used to compare groups against the No-Pre group.

\section{Results}

\subsection{Pre-exposure (Fig. 2A)}

Freezing levels during the pre-exposure session were low and did not differ between groups (freezing <3\% overall). Only three groups, $\mathrm{dCtx} \mathrm{A}_{\mathrm{n}=14}, \mathrm{dCtx} \mathrm{B} \mathrm{n}=14$, and Ctx $\mathrm{C}_{\mathrm{n}=6}$ received context pre-exposure. The No $\operatorname{Pre}_{\mathrm{n}}=6$ group did not receive context pre-exposure and was excluded from pre-exposure analysis. A significant main effect of time bin $[\mathrm{F}(1.928,124)=5.716, p<0.01]$ (Greenhouse-Geisser corrected) was detected. However, there was no main effect of group $[\mathrm{F}(2,31)=0.428, p>0.05]$ and no group $\mathrm{X}$ time bin interaction $[\mathrm{F}(3.856,124)=0.990, p>0.05]$ (Greenhouse-Geisser corrected). Exploratory post-hoc simple effects contrasts confirmed there were no differences at each time-point ( $p$ 's $>0.05)$.

In summary, visual images displayed on LCD screens did not induce significant freezing prior to conditioning.

\subsection{Testing (Fig. 2B)}

Freezing during the testing session differed between groups. A significant main effect of time bin $[\mathrm{F}(4,144)=3.109, p<0.05]$, a significant main effect of group $[\mathrm{F}(3,36)=3.668$, $p<0.05]$, and a significant group $\mathrm{X}$ time bin interaction $[\mathrm{F}(12,144)=1.885, p<0.05]$ were detected. Follow-up simple effects analyses contrasting group differences within each level combination of time-bin revealed that groups marginally differed at time bin one $[\mathrm{F}(3,36)=$ 2.581, $p=0.069]$, significantly differed at time bins two $[\mathrm{F}(3,36)=3.186, p<0.05]$, three $[\mathrm{F}(3,36)=3.532, p<0.05]$, and four $[\mathrm{F}(3,36)=3.799, p<0.05]$, and did not differ at time bin five $[\mathrm{F}(3,36)=0.148, p>0.05]$.

Post-hoc 2-sided Dunnet's test revealed that group dCtx A significantly differed from group No Pre at bins 2,3 , and 4 ( $p$ 's $<0.05$ ). dCtx B and Ctx C did not significantly differ from group No Pre ( $p$ 's $>0.05)$. Planned comparisons at time bins two, three, and four revealed the following: Group dCtx A significantly differed from group dCtx B at time bin $4[\mathrm{t}(26)=$ $2.088, p<0.05$ ], marginally at time bin 3 [ $\mathrm{t}_{\text {corrected }}(20.674)=2.006, p=0.058$ ], but not at time bin 2. Group dCtx A differed from group Ctx C at time bin $2\left[\mathrm{t}_{\text {corrected }}(16.50)=2.752\right.$, $p<0.05]$, and marginally at time bins 3 [ $\mathrm{t}(18)=-1.91, p=0.072]$, and 4 [ $\mathrm{t}(18)=-1.889, p$ $=0.075]$. Group dCtx A differed from group No Pre at time bins $2\left[\mathrm{t}_{\text {corrected }}(13.50)=\right.$ $-3.398, p<0.01], 3\left[\mathrm{t}_{\text {corrected }}(15.626)=-3.335, p<0.01\right]$, and $4\left[\mathrm{t}_{\text {corrected }}(14.747)=\right.$ $-3.458, p<0.01]$. Group dCtx B did not differ from Group Ctx C at any time bins ( $p$ 's $>$ $0.05)$. Group dCtx B differed from group No Pre at time bin $2\left[\mathrm{t}_{\text {corrected }}(14.347)=-2.922, p\right.$ $<0.05$ ], and marginally at time bins 3 [ $\left.\mathrm{t}_{\text {corrected }}(17.973)=-1.822, p=0.085\right]$, and 4 $\left[\mathrm{t}_{\text {corrected }}(18)=2.006, p\right]$. Grp Ctx C did not differ from Group No Pre at any time bin (p's > 
0.05). In summary, dCtx A significantly differed from immediate shock controls at time bins 2,3 , and 4 , but only differed from dCtx B at time bin 4 .

\section{Discussion}

In the present study, we asked if changes to visual information presented on LCD screens during context pre-exposure modulate subsequent conditioning to an immediate shock. Rats pre-exposed to the testing context (group dCtx A) demonstrated context conditioning in the CPFE relative to controls. Both control groups (No Pre and Ctx C) displayed the immediate shock deficit with little freezing during testing. In contrast, rats pre-exposed to an alternate visual image (group $\mathrm{dCtx}$ B) failed to show the CPFE, with freezing levels that only significantly differed from dCtx A at time bin 4 and group No Pre at time bin 2. Because rats from groups dCtx A and dCtx B were trained under identical contextual parameters (i.e., olfactory, tactile, visual, auditory, etc.), the differences in freezing are likely attributable to the visual images displayed during context pre-exposure. These data highlight how (1) visual information from an environment contributes to a portion of contextual fear learning and (2) LCD monitors can be incorporated into current fear conditioning setups to precisely control visual features.

During context pre-exposure, rats from each of the three pre-exposure groups displayed low levels of freezing, an indirect measure of context exploration. Towards the end of the preexposure session, freezing levels slightly increased across all groups likely reflecting habituation to the context (an indirect measure of context learning [37]). Importantly, preexposure freezing did not differ between groups. Taken together, these findings suggest that baseline freezing was not affected by (1) visual information presented via LCD screens or (2) by different images on the screens prior to conditioning.

During context testing, both $\mathrm{dCtx} \mathrm{A}$ and $\mathrm{dCtx} \mathrm{B}$ exhibited similar levels of freezing during the first minute. In the CPFE, the context memory associated with the immediate shock is that of the pre-exposure context [32]. It is possible that the $\mathrm{dCtx} \mathrm{B}$ group generalized fear to common features shared between the pre-exposure and testing environments during the initial moments of the retention test in $\mathrm{dCtx} \mathrm{A}$. However, freezing between groups $\mathrm{dCtx} \mathrm{A}$ and $\mathrm{dCtx} \mathrm{B}$ began to differ as the session progressed. Speculatively, this reduction in freezing over time may result from the sampling of additional features and visual information provided by the LCD screens. Future studies are needed to examine if testing in other contexts (e.g., dCtx B or Ctx C) produces a similar pattern of discrimination. Recent computational models of contextual fear predict this effect. For example, the contextual fear conditioning model developed by Krasne, Cushman, and Fanselow [19] predicts greater generalization between more familiar contexts. That is, contexts which share more features will initially produce high-levels of fear during testing, but this fear will quickly dissipate due to discrimination [19]. This model relies on the assumption that a limited number of features are sampled at any given moment and over time there is a re-evaluation of these features. Our data lend experimental support to this model. First, when few features were shared between the pre-exposure and testing contexts there was little generalization (i.e., group Ctx. C). However, when all but the visual features of the context were shared, the initially high levels of freezing rapidly dissipated. 
Given that contextual fear conditioning is believed to involve a composite representation of the multisensory experience [22], isolating the unique contributions of each feature in the environment - the tactile experience from the bars, the olfactory stimuli from cleaning agents, the spatial dimensions of the chamber, etc. - has proved challenging. Indeed, other studies have used gross manipulations of one or more sensory features to create distinct contexts e.g., [4,15,26,40,41]. Lee and colleagues [18] outline a number of limitations inherent to gross feature manipulations including vaguely defined contextual stimuli and a lack of within-session control. Our approach overcomes some of these limitations by providing the ability to precisely manipulate visual features within-session, thereby increasing experimental power over an important sensory feature during contextual fear conditioning. Future studies are needed to understand how within-session changes to visual features in the testing context impact defensive behaviors across different fear conditioning preparations (e.g., the CPFE paradigm, standard contextual fear conditioning, etc.) and to unconditioned visual stimuli (e.g., birds of prey; c.f. [8]).

It should be noted that Bucci and colleagues failed to show that rats could discriminate between two contexts (one paired with a foot-shock and one without) when only visual features were changed [4]. This is in agreement with other context discrimination studies showing that rats exhibit difficulty discriminating between highly similar (i.e., few feature differences) contexts. However, extended training (e.g., >14 days) can overcome this failure in discrimination between similar contexts [26]. In particular, the discrepancy between our results using the CPFE paradigm and Bucci et al. [4] may derive from procedural differences inherent to the behavioral paradigms. That is, context learning and context-shock learning occur on separate occasions in the CPFE paradigm, whereas these processes occur on the same occasion during discrimination learning. We would predict that manipulating visual features on LCD screens during context discrimination learning would replicate Bucci and colleagues (no discrimination over the first 10 days), but extended training (e.g., $>15$ days; [26]) would overcome this deficit. However, studies are needed to test these hypotheses.

It is important to note some key caveats with employing an LCD-based approach to fear conditioning preparations. First, we conducted our experiments using the Long-Evans strain of rat. Differences in fear conditioning (e.g., [7]) and innate freezing to predatory cues [30] have been reported between rat strains. Thus, it will be important for future studies to examine if our findings generalize to other strains or rodents. Due to the visual component of our design, it will also be necessary to account for differences in visual acuity e.g., Sprague-Dawley rats; $[27,28]$ between strains. Second, we detected our effects with a pumpkin vs. rock formation (see Fig. 1). We have not tested other images to exclude the possibility that our results are a selective phenomenon with these images or a general phenomenon of any images. However, other studies have applied alternative LCD-based methods to successfully manipulate spatial navigation $[1,14,16]$, discrimination learning [21], and defensive behaviors [8]. Finally, we only manipulated visual images during the pre-exposure phase in the CPFE paradigm - a paradigm which typically produces weaker conditioning under similar US parameters to single-trial contextual fear conditioning [35]. An examination of context discrimination using our approach (e.g., dCtx A+ [foot shock], $\mathrm{dCtx} \mathrm{B}-$ [no foot shock]) and during single-trial contextual fear conditioning are logical next steps. 
The present study offers a first step as a proof-of-concept in manipulating visual elements with LCD screens during incidental contextual learning in fear conditioning. More studies are needed to understand how rodents can visually discriminate between contexts in addition to how other elements [34] such as spatial [25], tactile, auditory, and olfactory [15] elements statistically contribute to contextual fear learning [12].

\section{Acknowledgements}

We thank Jamie Queensberry at the University of Delaware for help in designing and fabricating the chamber. We also thank the University of Delaware Office of Laboratory Animal Medicine for care of the animals. We would especially like to thank Mark E. Stanton and Jeffrey B. Rosen at the University of Delaware for their encouragement and support with this project.

Funding sources

This research was supported in part by the Oscar Kaplan Post-doctoral Fellowship in Developmental Issues awarded to Nathen J. Murawski with additional support to Arun Asok from 1 R01 HD075066-01A1 to ME Stanton and JB Rosen and the Department of Psychological and Brain Sciences at the University of Delaware.

\section{Abbreviations:}

CPFE

LCD

dCtx

AC

CS

US

Pre

No Pre

ANOVA context pre-exposure facilitation effect

liquid-crystal display

digital context

alternating current

conditional stimulus

unconditional stimulus

pre-exposure

no pre-exposure

analysis of variance

\section{References}

[1]. Aghajan ZM, Acharya L, Moore JJ, Cushman JD, Vuong C, Mehta MR,Impaired spatial selectivity and intact phase precession in two-dimensionalvirtual reality, Nat. Neurosci (2014).

[2]. Blanchard RJ, Blanchard DC, Crouching as an index of fear, J. Comp. Physiol. Psychol 67 (1969) 370. [PubMed: 5787388]

[3]. Bolles RC, Species-specific defense reactions and avoidance learning,Psychol. Rev 77 (1970) 32.

[4]. Bucci DJ, Saddoris MP, Burwell RD, Contextual fear discrimination isimpaired by damage to the postrhinal or perirhinal cortex, Behav. Neurosci 116 (2002) 479. [PubMed: 12049329]

[5]. Burman M, Murawski N, Schiffino F, Rosen J, Stanton M, Factors governingsingle-trial contextual fear conditioning in the weanling rat, Behav. Neurosci123 (2009) 1148. [PubMed: 19824781]

[6]. Cai DJ, Aharoni D, Shuman T, Shobe J, Biane J, Song W, Wei B, Veshkini M,La-Vu M, Lou J, A shared neural ensemble links distinct contextual memoriesencoded close in time, Nature 534 (2016) 115-118. [PubMed: 27251287]

[7]. Chang C.-h., Maren S, Strain difference in the effect of infralimbic cortexlesions on fear extinction in rats, Behav. Neurosci 124 (2010) 391. [PubMed: 20528083] 
[8]. De Franceschi G, Vivattanasarn T, Saleem AB, Solomon SG, Vision GuidesSelection of Freeze or Flight Defense Strategies in Mice, Current Biology, 2016.

[9]. Fanselow MS, Associative vs topographical accounts of the immediateshock-freezing deficit in rats: implications for the response selection rulesgoverning species-specific defensive reactions, Learn. Motiv 17 (1986) 16-39.

[10]. Fanselow MS, Contextual fear, gestalt memories, and the hippocampus, Behav. Brain Res 110 (2000) 73-81. [PubMed: 10802305]

[11]. Fanselow MS, Factors governing one-trial contextual conditioning, Anim. Learn. Behav 18 (1990) 264-270.

[12]. Fanselow MS, From contextual fear to a dynamic view of memory systems, Trends Cogn. Sci 14 (2010) 7-15. [PubMed: 19939724]

[13]. Fendt M, Fanselow MS, The neuroanatomical and neurochemical basis of conditioned fear, Neurosci. Biobehav. Rev 23 (1999) 743-760. [PubMed: 10392663]

[14]. Furtak SC, Cho CE, Kerr KM, Barredo JL, Alleyne JE, Patterson YR, Burwell RD, The floor projection maze: a novel behavioral apparatus forpresenting visual stimuli to rats, J. Neurosci. Methods 181 (2009) 82-88. [PubMed: 19422855]

[15]. González F, Quinn JJ, Fanselow MS, Differential effects of adding and removing components of a context on the generalization of conditional freezing, J. Exp. Psychol. Anim. Behav. Process 29 (2003) 78. [PubMed: 12561135]

[16]. Harvey CD, Collman F, Dombeck DA, Tank DW, Intracellular dynamics of hippocampal place cells during virtual navigation, Nature 461 (2009)941-946. [PubMed: 19829374]

[17]. Holland PC, Bouton ME, Hippocampus and context in classical conditioning,Curr. Opin. Neurobiol 9 (1999) 195-202. [PubMed: 10322181]

[18]. Kim S, Lee J, Lee I, The hippocampus is required for visually cued contextualresponse selection, but not for visual discrimination of contexts, Front. Behav. Neurosci 6 (2012) 66. [PubMed: 23060765]

[19]. Krasne FB, Cushman JD, Fanselow MS, A Bayesian context fear learning algorithm/automaton, Front. Behav. Neurosci 9 (2015) 112. [PubMed: 26074792]

[20]. LeDoux J, Emotion circuits in the brain, Annu. Rev. Neurosci 23 (2000) 155-184. [PubMed: 10845062]

[21]. Lee I, Shin JY, Medial prefrontal cortex is selectively involved in response selection using visual context in the background, Learn. Memory 19 (2012)247-250.

[22]. Maren S, Phan KL, Liberzon I, The contextual brain: implications for fearconditioning, extinction and psychopathology, Nat. Rev. Neurosci 14 (2013) 417-428. [PubMed: 23635870]

[23]. Matus-Amat P, Higgins EA, Barrientos RM, Rudy JW, The role of the dorsalhippocampus in the acquisition and retrieval of context memoryrepresentations, J. Neurosci 24 (2004) 2431-2439. [PubMed: 15014118]

[24]. Matus-Amat P, Higgins EA, Sprunger D, Wright-Hardesty K, Rudy JW, Therole of dorsal hippocampus and basolateral amygdala NMDA receptors in theacquisition and retrieval of context and contextual fear memories, Behav. Neurosci 121 (2007) 721. [PubMed: 17663597]

[25]. McHugh TJ, Tonegawa S, Spatial exploration is required for the formation of contextual fear memory, Behav. Neurosci 121 (2007) 335. [PubMed: 17469922]

[26]. Nakashiba T, Cushman JD, Pelkey KA, Renaudineau S, Buhl DL, McHugh TJ, Barrera VR, Chittajallu R, Iwamoto KS, McBain CJ, Young dentate granule cells mediate pattern separation, whereas old granule cells facilitate pattern completion, Cell 149 (2012) 188-201. [PubMed: 22365813]

[27]. Prusky GT, Harker KT, Douglas RM, Whishaw IQ, Variation in visual acuitywithin pigmented, and between pigmented and albino rat strains, Behav. Brain Res 136 (2002) 339-348. [PubMed: 12429395]

[28]. Prusky GT, West PWR, Douglas RM, Behavioral assessment of visual acuityin mice and rats, Vision Res 40 (2000) 2201-2209. [PubMed: 10878281]

[29]. Rashid AJ, Yan C, Mercaldo V, Hsiang H-LL, Park S, Cole CJ, De Cristofaro A, Yu J, Ramakrishnan C, Lee SY, Competition between engramsinfluences fear memory formation and recall, Science 353 (2016) 383-387. [PubMed: 27463673] 
[30]. Rosen JB, West EA, Donley MP, Not all rat strains are equal: differential unconditioned fear responses to the synthetic fox odor 2, 4, 5-trimethylthiazoline in three outbred rat strains, Behav. Neurosci 120 (2006) 290. [PubMed: 16719693]

[31]. Rudy J, Context representations, context functions, and the parahippocampal-hippocampal system, Learn. Memory 16 (2009) 573-585.

[32]. Rudy J, Barrientos R, O'Reilly R, Hippocampal formation supports conditioning to memory of a context, Behav. Neurosci 116 (2002) 530. [PubMed: 12148921]

[33]. Rudy J, Huff N, Matus-Amat P, Understanding contextual fear conditioning: insights from a twoprocess model, Neurosci. Biobehav. Rev 28 (2004)675-685. [PubMed: 15555677]

[34]. Rudy J, O’Reilly R, Contextual fear conditioning, conjunctive representations,pattern completion, and the hippocampus, Behav. Neurosci 113 (1999) 867. [PubMed: 10571471]

[35]. Schreiber WB, Asok A, Jablonski SA, Rosen JB, Stanton ME, Egr-1 mRNA expression patterns in the prefrontal cortex, hippocampus, and amygdala during variants of contextual fear conditioning in adolescent rats, Brain Res1576 (2014) 63-72. [PubMed: 24976583]

[36]. Smith DM, Bulkin DA, The form and function of hippocampal context representations, Neurosci. Biobehav. Rev 40 (2014) 52-61. [PubMed: 24462752]

[37]. Stote DL, Fanselow MS, NMDA receptor modulation of incidental learning in Pavlovian context conditioning, Behav. Neurosci 118 (2004) 253. [PubMed: 14979804]

[38]. Swan AA, Clutton JE, Chary PK, Cook SG, Liu GG, Drew MR, Characterization of the role of adult neurogenesis in touch-screen discrimination learning, Hippocampus 24 (2014) 1581-1591. [PubMed: 25074617]

[39]. Thomas BB, Samant DM, Seiler MJ, Aramant RB, Sheikholeslami S, Zhang K, Chen Z, Sadda $\mathrm{SR}$, Behavioral evaluation of visual function of rats using a visual discrimination apparatus, J. Neurosci. Methods 162 (2007)84-90. [PubMed: 17289151]

[40]. Wang S-H, Teixeira CM, Wheeler AL, Frankland PW, The precision of remote context memories does not require the hippocampus, Nat. Neurosci12 (2009) 253-255. [PubMed: 19182794]

[41]. Wiltgen BJ, Zhou M, Cai Y, Balaji J, Karlsson MG, Parivash SN, Li W, Silva AJ, The hippocampus plays a selective role in the retrieval of detailed contextual memories, Curr. Biol 20 (2010) 1336-1344. [PubMed: 20637623] 


\section{HIGHLIGHTS}

- $\quad$ Features of a context may differentially contribute to the CS-US representation in contextual fear conditioning.

- Visual information from an environment may statistically contribute to contextual fear learning.

- $\quad$ LCD monitors can be readily incorporated to current fear conditioning protocols in order to precisely control visual stimuli of contexts. 
A

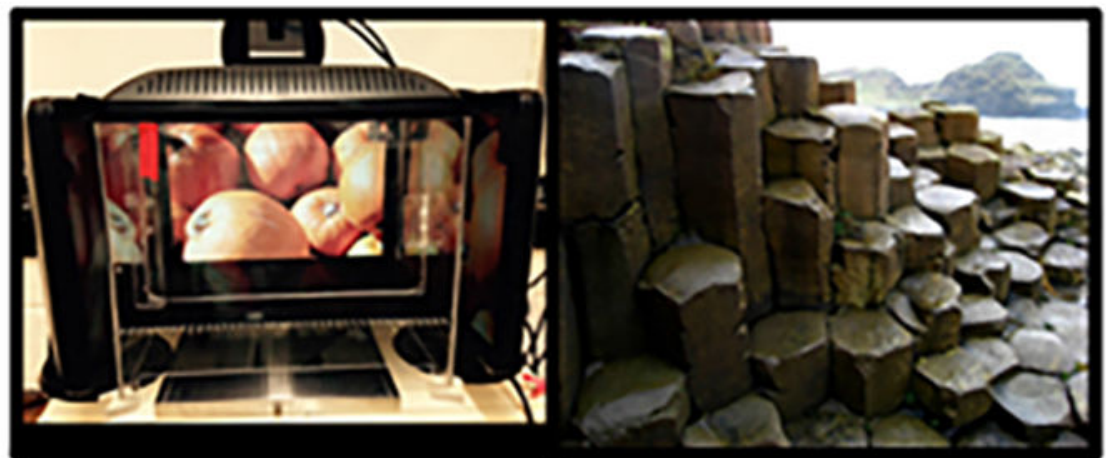

Digital Context A

Digital Context B

B

Pre-exposure

Training

Testing

$\mathrm{dCtxA}$

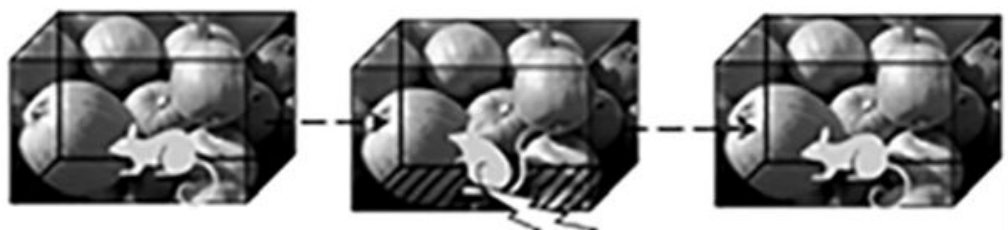

dCtx B

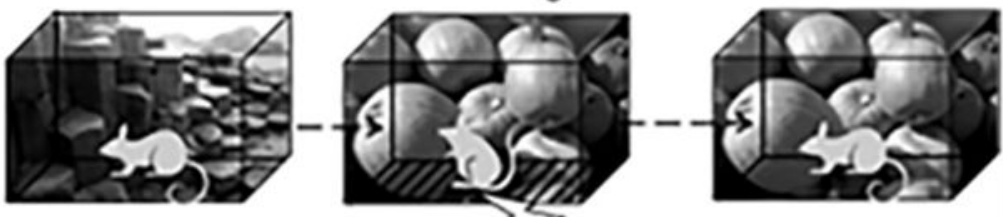

$\operatorname{Ctx} \mathrm{C}$

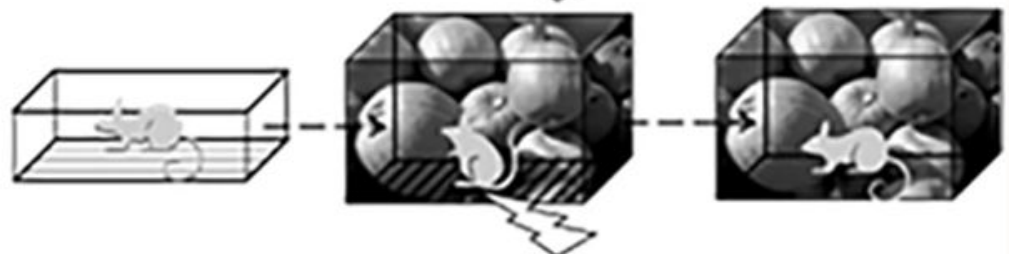

No Pre

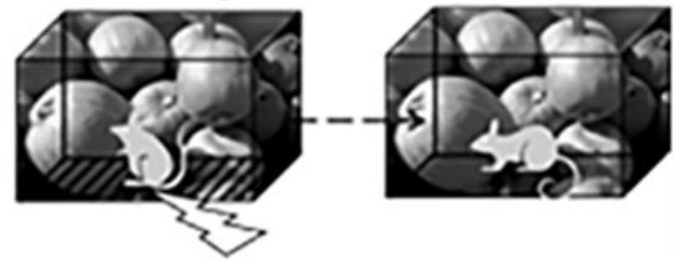

Fig. 1.

Visual Images and CPFE Behavioral Design. (A) Image of the conditioning apparatus with LCD screens and the visual images used for digital context (dCtx) A (left panel) and dCtx B (right panel). dCtx A and dCtx B were identical except for either pumpkins or a rock formation displayed on the LCD screens. Ctx C was distinct with regard to spatial, tactile, visual, olfactory, and auditory characteristics. No Pre animals were not pre-exposed to any context. (B)The CPFE fear conditioning experiments were run over three days. On day 1, rats were pre-exposed to $\mathrm{dCtx} \mathrm{A}, \mathrm{dCtx} \mathrm{B}, \mathrm{CtxC}$, or remained in their home cages (No Pre). 
On day 2, all groups were given immediate shock training in $\mathrm{dCtx}$ A. On day 3 , all groups were tested for conditioned freezing in $\mathrm{dCtx} \mathrm{A}$. 


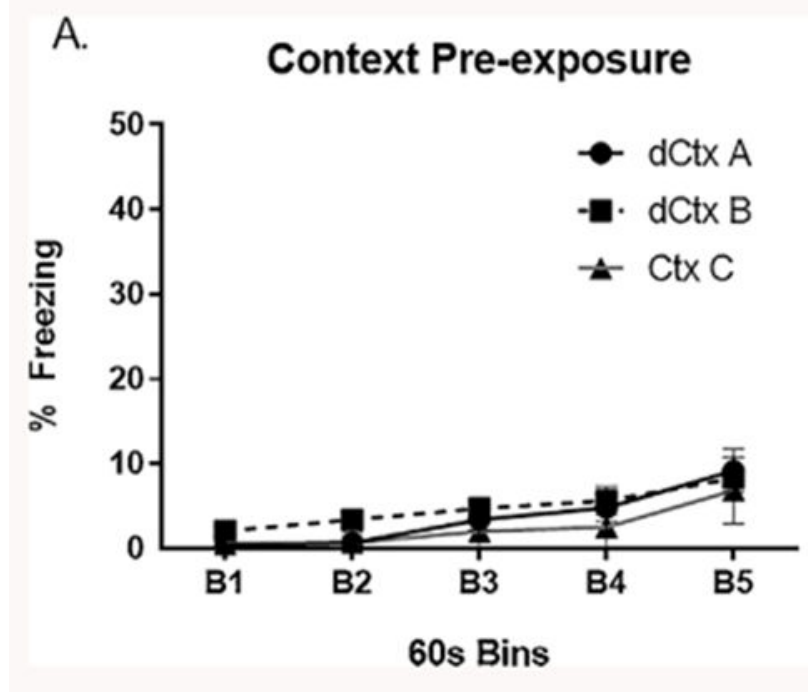

B.

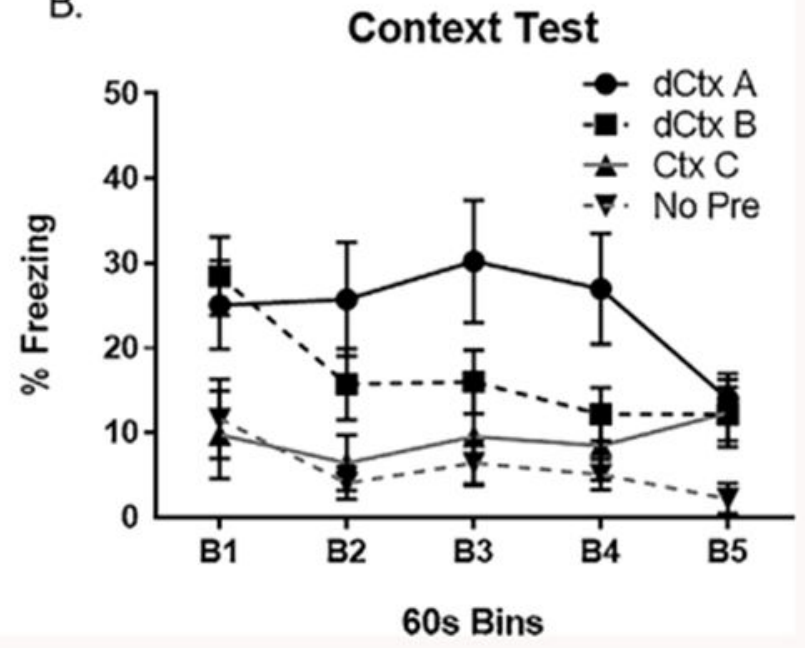

Fig. 2.

Freezing during context pre-exposure and testing. Rats were pre-exposed to digital Context A ( $\mathrm{dCtx} A)$, digital Context B ( $\mathrm{dCtx} B)$, or an alternate context (Ctx C). (A) The percentage of time rats froze during the $300 \mathrm{~s}$ context pre-exposure session. All groups showed low levels of freezing during context pre-exposure with no differences between groups. (B) During the context test, rats pre-exposed to dCtx A showed significantly higher levels of freezing across the $300 \mathrm{~s}$ testing session relative to rats without context pre-exposure. Rats pre-exposed to dCtx B did not highly differ from controls. 